\title{
Performance analysis of a multi-function photonic architecture on SOI acting as a frequency shifter
}

\author{
Gazi Mahamud Hasan ${ }^{1 *}$, Mehedi Hasan ${ }^{1}$, Trevor Hall ${ }^{1}$ \\ ${ }^{1}$ Photonic Technology Laboratory, Centre for Research in Photonics, \\ University of Ottawa \\ Ottawa, Canada \\ *ghasa102@uottawa.ca
}

\begin{abstract}
:
A multi-functional photonic circuit implemented on silicon on insulator (SOI) platform is reported. Eight phase modulators are connected to provide four Mach-Zehnder interferometer structure in parallel. Multimode interference couplers are used as the splitter/combiner of the MZI structures to provide static DC bias. This architecture generates two principle spatially separated $1^{\text {st }}$ order harmonics from a single light source, which may be collected from separate ports without using any optical demultiplexing filters which permits remote heterodyning. A carrier suppression of $>20 \mathrm{~dB}$ and spurious sideband suppression $>$ $12 \mathrm{~dB}$ relative to the principle harmonics is achieved with bias voltage tuning only. In addition to the frequency conversion, the circuit can also perform as a sub-carrier generator, IQ modulator and frequency multiplier.
\end{abstract}

\section{Introduction:}

The ever-growing data traffic and super broadband services demanded by the end users have led the wireless communication network to undergo rapid development in terms of capacity, bandwidth, cost and mobility. The low-power, mature lower frequency bands are already congested and offer small capacity in wireless communication. On the other hand, the high capacity high frequency wireless system is inherently power hungry, costly and imposes complicated coverage schemes. This bottleneck has introduced the radio-overfiber (RoF) system as a viable solution for broadband wireless access networks [1-2]. RoF technology can exploit the low loss, lower power consumption and broad bandwidth offered by optical fiber communication by distributing the radio signals over the optical fiber. Traditionally, RoF employs analogue intensitymodulated direct detection (IM-DD) optical links [3-4] which is prone to high power consumption, limited dynamic range and periodic RF power fading. Hall et al. reasoned that broadband wireless access network using distributed antenna system architecture deployed in cluttered urban environments complemented by a digital coherent optical RoF link provides an energy efficient solution [5]. The millimeter-wave (mmwave) carrier generation at downlink and efficient single sideband (SSB) modulation at uplink are two major factors to be considered for improvement of digital coherent optical transport of RF signal [6-8]. Numerous researches have been undertaken on photonic mm-wave generation techniques. The simplest approach is the heterodyne beating of two phase correlated optical carriers separated in frequency at a high speed photo-detector. The conventional methods are based on a dual-mode laser [9], a dual lasers with phase locking [10-11] or mode-lock lasers [12] which all suffer low RF coherency or limited tunability. External modulation based photonic mm-wave generation techniques are emerging as a feasible candidate due to its higher spectral purity, simplicity, stability and low RF frequency requirement [13-15]. A common feature of most external modulation based architectures is that the pair of high-order harmonics separated by the desired RF carrier frequency is emitted from the same output port. Hence, optical frequency selective components such as arrayed wavelength grating (AWG), frequency de-multiplexer are needed to separate these sub-carriers in order that they may be modulated individually. This introduces complexity in system implementation in terms of tuning, selectivity and temperature sensitivity [16-17].

Yamazaki et al. demonstrated that a complementary frequency shifter capable of spatial frequency separation can be implemented and integrated along with an in-phase quadrature-phase (IQ) modulator to 
obtain separate subcarrier modulation without using any optical frequency de-multiplexer and achieved up to a data rate of $400 \mathrm{Gbit} / \mathrm{s}$ using dual-polarization (DP) 16-level QAM [18-19]. A digital coherent RoF system with downlink utilizing a higher order SSB modulator as a frequency shifter to generate mm-wave has been proposed by Hassan \& Hall [20]. Their architecture exploits the spatial separation of higher order sideband for frequency multiplication and consequently can be operated in a low RF frequency regime.

The application of a photonic frequency shifter is not limited to a heterodyne light communication system. The other sectors where it plays a key role are laser Doppler velocimetry (LDV) and Doppler Lidar systems [21-22], high quality multicarrier generation with a recirculating frequency shifting loop [23-24], frequency-shifted feedback (FSF) laser [25], true time delay beam steering in frequency domain continuous wave (FMCW) radars [26], high-resolution laser spectroscopy [27] and measurements of ultra-high-Q photonic crystal nanocavities [28]. Various techniques have been pursued to realize a high-performance frequency shifter. Early photonic frequency shifters are based on discrete components [29-31]. To the best of the authors' knowledge, an integrated frequency shifter based on a titanium-diffused $\mathrm{LiNbO}_{3}$ optical waveguide circuit was reported for the first time in 1981 [32]. The most conventional method to obtain frequency conversion is to use a OSSB modulator followed by filter structure to suppress carrier and/or unwanted sideband [33-36]. Its operation is limited by the frequency response of the filter. In addition, the optical source, modulator and the filter must be very closely matched in frequency of operation that involves temperature control, which is undesirable in terms of energy consumption.

Mach-Zehnder interferometer (MZI) based frequency conversion has been investigated intensely [32, 3740]. Carrier and unwanted sideband suppression by design is possible by applying appropriate RF signals with strict relative phase relationship, biasing the MZI structure to proper operating point and/or implementing phase-shift elements within the arms of a MZI. The spectral purity can be impaired by any imbalance in the mentioned features. Mature $\mathrm{LiNbO}_{3}$ modulator technology is a natural choice for frequency shifting purpose as its extinction ratio is relatively high and it can offer a linear electro-optic effect with large bandwidth. However, it needs high drive voltage, suffers from severe dc bias drift caused by charge screening and dielectric relaxation and is not suitable for large-scale photonic integration. Compact footprint can be gained by using the Si platform which can exploit CMOS compatible industry standards for optoelectronic integration. With the recent studies on high-speed modulator, Si optical frequency converters with excellent performance are emerging. Lauermann et al. demonstrated an integrated frequency shifter on a Silicon-Organic Hybrid (SOH) platform with conversion efficiency of $5.8 \mathrm{~dB}$ while suppressing spurious sidebands by more than $23 \mathrm{~dB}$ for a frequency range up to $10 \mathrm{GHz}$ [41]. Another Si photonic frequency shifter has been reported recently with carrier suppression of $27 \mathrm{~dB}$ and at least $12 \mathrm{~dB}$ sideband suppression at $1 \mathrm{GHz}$ [42].

Other methods for frequency conversion includes traveling wave TE/TM mode conversion [43], serrodyne translation [44-45] and acousto-optic deflection [30-31, 46-47]. Serrodyne technique can offer high carrier and spurious sideband suppression when a sawtooth shaped modulating signal with very small fall time (very small percentage of the sawtooth signal period) can be applied. This implies a very high bandwidth for the modulator and driving circuit are needed for sufficient spectral purity and high achievable frequency shift. In addition, a conventional Si diode modulator cannot be operated with serrodyne signal and so, thermo-optic modulation is necessary, which restricts its high frequency operation. Acousto-optic modulation depends on the acoustic wave induced refractive index variation which demands delicate phase matching. The operation is inherently limited to low frequency and, as Si is not piezoelectric hybridization with other material becomes mandatory. All-optical frequency shifting operations based on stimulated Brillouin scattering in optical fibers has also been demonstrated [48-49], which are limited by the fixed Brillouin frequency of a fiber and a complicated pump wave locking procedure.

A multi-functional photonic circuit consisting of four differentially driven MZMs in parallel has been proposed in [50]. Sub-carrier generation, frequency multiplication, complex modulation and frequency 
conversion can be achieved by the same architecture. Each functionality is associated with a combination of MZM biasing, optical phase shifting and RF relative phase relationship which can be obtained by outside control. Table 1 describes the conditions for achieving these function. Figure 2 shows the schematic of the proposed circuit. In this report, we demonstrate the frequency shifting capability of the proposed architecture [50]. The silicon-on-insulator (SOI) platform has been used for the fabrication of the circuit. Multimode interference (MMI) couplers are used as the splitter/combiner of the MZI structures to avoid DC bias drift problem. Besides, MMI offers wideband operation, less sensitivity to slight fabrication defects and low loss. The circuit had been fabricated to prove its implementation feasibility and so, to avoid complexity, tuning mechanism had been avoided in the design. The observations obtained from our experiments is encouraging for future fabrication with proper tuning mechanism and implementation in the digital coherent RoF link of a viable sub-carrier generator with higher order frequency multiplication at downlink and SSB modulator at uplink.

Table 1: List of parameters necessary to obtain target application (MITP: Maximum transmission point; MITP: Minimum transmission point; Complex signal $=V_{I}+i V_{Q}$ )

\begin{tabular}{|c|c|c|c|c|c|}
\hline & & $\begin{array}{c}\text { Sub-carrier } \\
\text { generation }\end{array}$ & $\begin{array}{c}\text { Frequency } \\
\text { conversion/SSB } \\
\text { modulation }\end{array}$ & $\begin{array}{c}\text { IQ } \\
\text { modulation }\end{array}$ & Frequency 8-tupling \\
\hline \multirow{4}{*}{$\begin{array}{c}\text { MZM DC } \\
\text { bias point }\end{array}$} & $\mathrm{MZM}_{\mathrm{A}}$ & MITP & MITP & MITP & MATP \\
\cline { 2 - 6 } & $\mathrm{MZM}_{\mathrm{B}}$ & MITP & MITP & MITP & MATP \\
\cline { 2 - 6 } & $\mathrm{MZM}_{\mathrm{C}}$ & MITP & MITP & MITP & MATP \\
\cline { 2 - 6 } & $\mathrm{MZM}_{\mathrm{D}}$ & MITP & MITP & MITP & MATP \\
\hline \multirow{4}{*}{$\begin{array}{c}\text { Optical phase } \\
\text { shift }\end{array}$} & $\mathrm{MZM}_{\mathrm{A}}$ & $\pi / 4$ & 0 & 0 & 0 \\
\cline { 2 - 6 } & $\mathrm{MZM}_{\mathrm{B}}$ & 0 & 0 & 0 & 0 \\
\cline { 2 - 6 } & $\mathrm{MZM}_{\mathrm{C}}$ & $2 \pi / 4$ & 0 & 0 & 0 \\
\cline { 2 - 6 } & $\mathrm{MZM}_{\mathrm{D}}$ & $3 \pi / 4$ & $V_{R F} \sin \left(\omega_{R F} t\right)$ & $V_{I}$ & $V_{R F} \cos \left(\omega_{R F} t\right)$ \\
\hline \multirow{3}{*}{\begin{tabular}{c} 
sodulating \\
\cline { 2 - 6 }
\end{tabular}} & $\mathrm{MZM}_{\mathrm{A}}$ & $V_{R F} \sin \left(\omega_{R F} t\right)$ & $V_{R F} \sin \left(\omega_{R F} t+\pi / 2\right)$ & $V_{Q}$ & $V_{R F} \cos \left(\omega_{R F} t+\pi / 4\right)$ \\
\cline { 2 - 6 } & $\mathrm{MZM}_{\mathrm{B}}$ & $V_{R F} \sin \left(\omega_{R F} t+\pi / 4\right)$ & $V_{R F} \sin \left(\omega_{R F} t+\pi / 2\right)$ & $V_{Q}$ & $V_{R F} \cos \left(\omega_{R F} t+\pi / 2\right)$ \\
\cline { 2 - 6 } & $\mathrm{MZM}_{\mathrm{C}}$ & $V_{R F} \sin \left(\omega_{R F} t+3 \pi / 4\right)$ & $V_{R F} \sin \left(\omega_{R F} t\right)$ & $V_{I}$ & $V_{R F} \cos \left(\omega_{R F} t+3 \pi / 4\right)$ \\
\cline { 2 - 6 } & $\mathrm{MZM}_{\mathrm{D}}$ & $V_{R F} \sin \left(\omega_{R F} t+2 \pi / 4\right)$ & & &
\end{tabular}

\section{Theory:}

The conventional waveguide-based method for frequency shifting utilizing single sideband modulation (SSB) by an in-phase/quadrature (IQ) modulator was first described by Izutsu et al. [32]. Their architecture consists of a dual parallel Mach-Zehnder modulator (DPMZM) which has two differential MZMs, each on one arm of an outer Mach-Zehnder interferometer (MZI) structure. The MZMs are set to their minimum

(a)

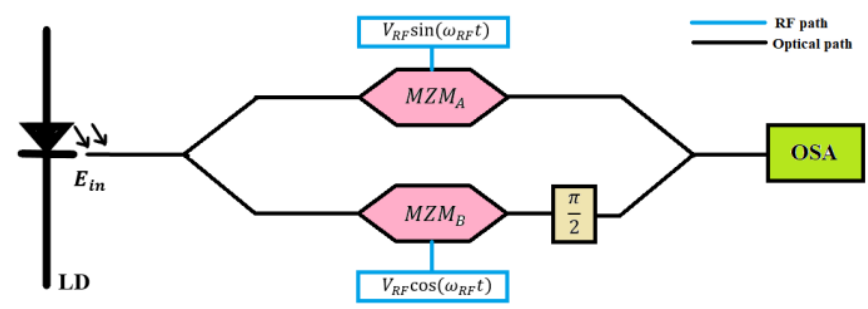

(b)

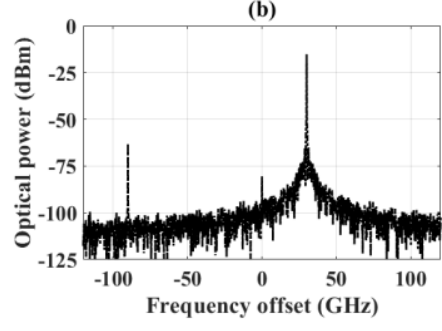

Figure 1: (a) Schematic diagram of conventional IQ modulator based optical frequency shifter. The MZMs are differentially driven. $\pi / 2$ optical phase shift is applied to bias the outer MZI to its quadrature point (b) Optical spectrum of the corresponding circuit showing frequency shifting of the carrier by the RF frequency $30 \mathrm{GHz}$. Simulation is done using Virtual photonics Inc. (VPI) software package. MZM: Mach-Zehnder modulator, OSA: Optical spectrum analyzer, LD: Laser diode 
transmission bias points and act as amplitude modulators. The outer MZI is set to its quadrature bias point i.e. one arm has a phase shift of $\pi / 2$ with respect to the other. Figure 1 shows the architecture and the output optical spectrum of this IQ modulator.

The circuit shown in figure 1(a) can perform the SSB frequency electro-optical up-conversion function if an RF electrical signal is applied to the I channel and a $\pi / 2$ phase-shifted replica of the same RF electrical signal is applied to the $\mathrm{Q}$ channel. In the ideal situation, each of the Y-splitters has a perfect 3-dB splitting ratio which leads to the transmission function of the circuit to be:

$$
\begin{gathered}
f_{1}(t)=\frac{i}{2}\left\{\sin \left[m \cos \left(\omega_{R F} t\right)\right]+i \sin \left[m \sin \left(\omega_{R F} t\right)\right]\right\} \\
\Rightarrow \\
f_{1}(t)=i\left\{J_{1}(m) \exp \left[j \omega_{R F} t\right]-J_{3}(m) \exp \left[-j 3 \omega_{R F} t\right] \ldots\right\}
\end{gathered}
$$

where $m=\pi V_{\mathrm{RF}} / v_{\pi}$ is the modulation index, $v_{\pi}$ is the half-wave voltage of the modulator, $J_{p}$ is the Bessel function of the first kind of order $p$ and $\omega_{R F}$ is the RF angular frequency. The optical spectrum shown in figure 1(b) is achieved for $V_{\mathrm{RF}}=0.1 v_{\pi}$. Restricting operation in small signal modulation range results in lower conversion efficiency but high side-harmonic suppression [41]. A polarity change at the phase in the outer MZI can switch the upper sideband (USB) to lower sideband (LSB) operation.

Maldonado-Basilio et al. proposed an electro-optical up-conversion mixer which utilizes the intrinsic relative phase relationships between the ports of MMI coupler to provide the necessary static phase, eliminating the need of static bias, static phase element and trimming [51]. Another photonic frequency multiplier circuit uses the same concept of exploiting the phase relationship of input and output signals in the MMI coupler to obtain an 8-fold multiplication of the electric local oscillator frequency [52-53]. Mehedi Hassan et al. extended the circuits functionality to have dual-carrier complex modulation, sub-carrier generation and frequency up-conversion [50]. This photonic circuit consists of eight phase modulator situated in the arms between an interconnected $4 \times 8$ distribution tree and a complementary $8 \times 4$ combination tree. The $4 \times 8$ distribution tree starts with an outer stage of $4 \times 4$ MMI coupler which is followed by an inner stage consisting of two parallel pairs of $2 \times 2$ MMI couplers. The $8 \times 4$ combination tree maintains a reciprocal structure of the distribution tree. Four MZI structures can be formed by connecting each pair of phase modulators to the inner stage MMIs as shown in figure 2(a). Each MZI configuration can act as an MZM when differentially driven. A little modification in the connections between the outer and inner stages at the distribution and combination sides which would translate into the bias point reversal converts the frequency 8-tupling circuit in [52] to the IQ modulator in [50]. The schematic diagram of the modified IQ modulator is shown in figure 2 (b).

To achieve the IQ modulator operation, each MZM is to be biased at its minimum transmission point (MITP). This can be maintained by selecting appropriate input and output ports of each MZI which offers the necessary static optical phase difference between the arms of MZM due to the intrinsic relative phase relationships between the ports of MMI coupler. Figure 2(a) shows the choice of input-output ports for which MITP condition for each MZM can be achieved by design. The transfer matrix of individual MZM can be expressed as:

$$
\left[\begin{array}{l}
b_{1} \\
b_{2}
\end{array}\right]=T_{2 \times 2}\left[\begin{array}{cc}
\exp \left(i \varphi_{1}\right) & 0 \\
0 & \exp \left(i \varphi_{2}\right)
\end{array}\right] T_{2 \times 2}\left[\begin{array}{l}
a_{1} \\
a_{2}
\end{array}\right]
$$

where $T_{2 \times 2}=\frac{1}{\sqrt{2}}\left[\begin{array}{cc}1 & -i \\ -i & 1\end{array}\right]$ is the transfer matrix of a $2 \times 2$ MMI coupler and $\varphi_{n}$ is the phase shift applied to the $\mathrm{n}^{\text {th }}$ arm of an MZM. Based on the input/ output ports of each MZM chosen and the phase shift applied to drive each MZM differentially, the transmission function of each MZM can be obtained as: 
(a)

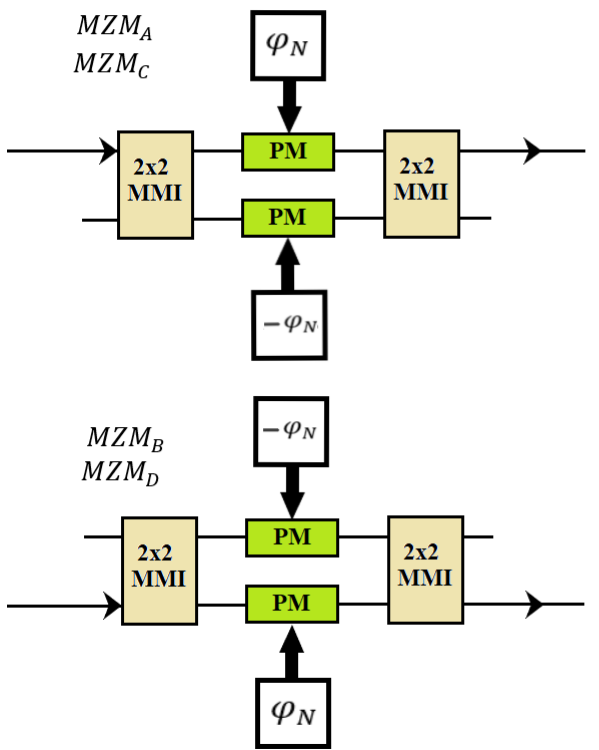

(b)

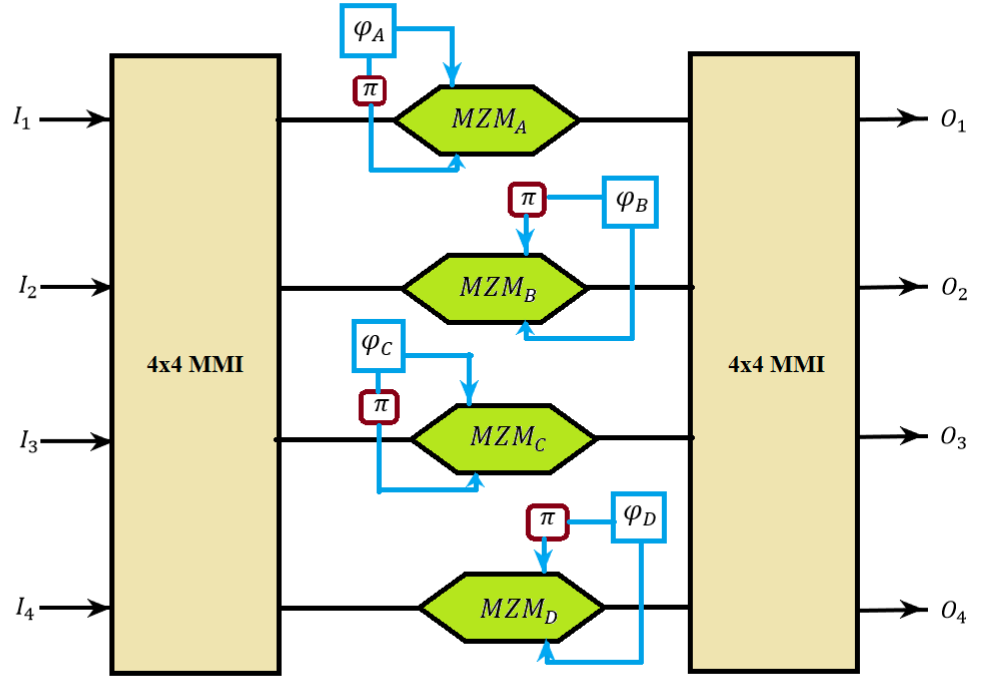

Figure 2: (a) Schematic of the differentially driven MZMs; (b) Schematic diagram of the proposed frequency shifter circuit. Each MZM is driven differentially with modulating signals $\varphi_{A}, \varphi_{B}, \varphi_{C}$ and $\varphi_{D}$ respectively. MMI: Multimode interference coupler, PM: Phase modulator, MZM: Mach-Zehnder modulator

$$
f_{M Z M_{N}}=i \sin \left(\varphi_{N}\right)
$$

The four parallel MZMs biased at MITP provide the framework of two parallel IQ modulators. The necessary optical phase difference between two MZMs of each IQ modulator can be obtained by a proper interconnection between the inner MZM stage and the $4 \times 4$ MMI couplers at the outer stages. The detailed schematic diagram in figure 2(b) shows a mirror symmetry in terms of circuit connection. Several ways for the application of RF inputs satisfying the condition of biasing the outer MZI of each IQ modulator at its quadrature point are possible. Figure 2(b) shows one example which retains the mirror symmetry. In this architecture, the upper two MZMs form one IQ modulator and the lower two MZMs form the other one.

The total transfer matrix of the architecture can be expressed as [52]:

$$
\left[\begin{array}{l}
O_{1} \\
O_{2} \\
O_{3} \\
O_{4}
\end{array}\right]=i T_{4 \times 4}\left[\begin{array}{cccc}
\sin \left(\varphi_{A}\right) & 0 & 0 & 0 \\
0 & \sin \left(\varphi_{B}\right) & 0 & 0 \\
0 & 0 & \sin \left(\varphi_{C}\right) & 0 \\
0 & 0 & 0 & \sin \left(\varphi_{D}\right)
\end{array}\right] T_{4 \times 4}\left[\begin{array}{c}
I_{1} \\
I_{2} \\
I_{3} \\
I_{4}
\end{array}\right]
$$

where $T_{4 \times 4}=\frac{1}{\sqrt{4}}\left[\begin{array}{rrrr}1 & -\zeta & \zeta & 1 \\ -\zeta & 1 & 1 & \zeta \\ \zeta & 1 & 1 & -\zeta \\ 1 & \zeta & -\zeta & 1\end{array}\right]$ is the transfer matrix of a $4 \times 4$ MMI coupler. Here, $\zeta=e^{i \pi / 4}$.

Assuming only $I_{1}$ to be connected to the optical source and two modulating signals $V_{1}=V_{I 1}+i V_{Q 1}$ and $V_{2}=V_{I 2}+i V_{Q 2}$ to be applied in such a way that $\varphi_{A}=\pi V_{I 1} / v_{\pi}, \varphi_{B}=\pi V_{Q 1} / v_{\pi}, \varphi_{C}=\pi V_{Q 2} / v_{\pi}$ and $\varphi_{D}=\pi V_{I 2} / v_{\pi}$, the outputs of the architecture can be expressed as:

$$
\left[\begin{array}{l}
O_{1} \\
O_{2} \\
O_{3} \\
O_{4}
\end{array}\right]=\frac{i}{4}\left[\begin{array}{c}
\sin \left(\pi V_{I 1} / v_{\pi}\right)+i \sin \left(\pi V_{Q 1} / v_{\pi}\right)+\sin \left(\pi V_{I 2} / v_{\pi}\right)+i \sin \left(\pi V_{Q 2} / v_{\pi}\right) \\
\zeta\left\{\sin \left(\pi V_{I 1} / v_{\pi}\right)-\sin \left(\pi V_{Q 1} / v_{\pi}\right)+\sin \left(\pi V_{I 2} / v_{\pi}\right)+\sin \left(\pi V_{Q 2} / v_{\pi}\right)\right\} \\
\left.\sin \left(\pi V_{I 1} / v_{\pi}\right)-\sin \left(\pi V_{Q 1} / v_{\pi}\right)-\sin \left(\pi V_{I 2} / v_{\pi}\right)+\sin \left(\pi V_{Q 2} / v_{\pi}\right)\right\} \\
\sin \left(\pi V_{Q 1} / v_{\pi}\right)+\sin \left(\pi V_{I 2} / v_{\pi}\right)-i \sin \left(\pi V_{Q 2} / v_{\pi}\right)
\end{array}\right]\left[I_{1}\right]
$$


For $\left(V_{I 1} / v_{\pi}, V_{Q 1} / v_{\pi}, V_{I 2} / v_{\pi}, V_{Q 2} / v_{\pi}\right) \ll 1$, equation (5) can be written as:

$$
\left[\begin{array}{l}
O_{1} \\
O_{2} \\
O_{3} \\
O_{4}
\end{array}\right]=\frac{i \pi}{4 v_{\pi}}\left[\begin{array}{c}
\zeta\left\{\left(V_{I 2}-V_{I 1}\right)+\left(V_{Q 2}-V_{Q 1}\right)\right\} \\
\zeta\left\{\left(V_{I 1}-V_{I 2}\right)+\left(V_{Q 2}-V_{Q 1}\right)\right\} \\
V_{1}{ }^{*}+V_{2}{ }^{*}
\end{array}\right]\left[I_{1}\right]
$$

where (*) stands for complex conjugate. In the case of a pure tone modulating signal, i.e. $\varphi_{A}=\varphi_{D}=$ $m \cos \left(\omega_{R F} t\right)$ and its companion $\pi / 2$ phase shifted replica, i.e. $\varphi_{B}=\varphi_{C}=m \cos \left(\omega_{R F} t+\pi / 2\right)$, the outputs of the architecture can be expressed as:

$$
\begin{aligned}
{\left[\begin{array}{l}
O_{1} \\
O_{2} \\
O_{3} \\
O_{4}
\end{array}\right]=} & {\left[\begin{array}{c}
-i \sum_{n=1}^{\infty} J_{2 n-1}(m)\left\{(-1)^{n} \cos \left[(2 n-1) \omega_{R F} t\right]+i \sin \left[(2 n-1) \omega_{R F} t\right]\right\} \\
0 \\
0 \\
-i \sum_{n=1}^{\infty} J_{2 n-1}(m)\left\{(-1)^{n} \cos \left[(2 n-1) \omega_{R F} t\right]-i \sin \left[(2 n-1) \omega_{R F} t\right]\right\}
\end{array}\right]\left[I_{1}\right] } \\
\Rightarrow & =\left[\begin{array}{c}
O_{1} \\
O_{2} \\
O_{3} \\
O_{4}
\end{array}\right]=\left[\begin{array}{c}
i\left\{J_{1}(m) \exp \left[-j \omega_{R F} t\right]-J_{3}(m) \exp \left[j 3 \omega_{R F} t\right] \ldots\right\} \\
0 \\
0 \\
i\left\{J_{1}(m) \exp \left[j \omega_{R F} t\right]-J_{3}(m) \exp \left[-j 3 \omega_{R F} t\right] \ldots\right\}
\end{array}\right]\left[I_{1}\right]
\end{aligned}
$$

It can be observed from equation (7) that for this specific input/output combination and local oscillator setting, this photonic architecture performs an electrical to optical frequency up-conversion with the lower optical sideband available from output port $O_{1}$ and the upper optical sideband available from output port $\mathrm{O}_{4}$. In principle, all even order harmonics, including the carrier are suppressed. Among the odd order harmonics, for output port $O_{1}$, positive orders equal to $(2 \mathrm{p}+1)$, with $\mathrm{p}$ even, and negative orders equal to $-(2 \mathrm{p}+1)$, with $\mathrm{p}$ odd, are suppressed and vice versa for output port $O_{4}$. By energizing other input ports individually, the LSB-USB operations shift to other output ports. Interchanging the local oscillator setting, i.e. $\varphi_{A}=\varphi_{D}=m \cos \left(\omega_{R F} t+\pi / 2\right)$ and $\varphi_{B}=\varphi_{C}=m \cos \left(\omega_{R F} t\right)$ leads the LSB-USB operation to swap their respective output ports, maintaining their distinct spatial separation.

It is possible to remove one IQ modulator completely from the operation by disconnecting the RF local oscillators to it. For equal phase shift at both arms of an MZI architecture, it will act as a cross-over if there is no external perturbation in phase. From figure 2, it can be observed that all the cross-ports are unused which means that any MZI can be cut-off completely at the expense of optical power. Taking $\varphi_{C}=\varphi_{D}=$ 0 leads the removal of the lower IQ modulator to be from the end-to-end optical path which modifies equation (4) to:

$$
\begin{aligned}
& {\left[\begin{array}{l}
O_{1} \\
O_{2} \\
O_{3} \\
O_{4}
\end{array}\right]=i T_{4 \times 4}\left[\begin{array}{cccc}
\sin \left(\varphi_{A}\right) & 0 & 0 & 0 \\
0 & \sin \left(\varphi_{B}\right) & 0 & 0 \\
0 & 0 & \sin \left(\varphi_{C}\right) & 0 \\
0 & 0 & 0 & \sin \left(\varphi_{D}\right)
\end{array}\right] T_{4 \times 4}\left[I_{1}\right]} \\
& {\left[\begin{array}{l}
O_{1} \\
O_{2} \\
O_{3} \\
O_{4}
\end{array}\right]=\frac{1}{2}\left[\begin{array}{c}
-i \sum_{n=1}^{\infty} J_{2 n-1}(m)\left\{(-1)^{n} \cos \left[(2 n-1) \omega_{R F} t\right]+i \sin \left[(2 n-1) \omega_{R F} t\right]\right\} \\
e^{i 3 \pi / 4} \sum_{n=1}^{\infty} J_{2 n-1}(m)\left\{(-1)^{n} \cos \left[(2 n-1) \omega_{R F} t\right]+\sin \left[(2 n-1) \omega_{R F} t\right]\right\} \\
-e^{i 3 \pi / 4} \sum_{n=1}^{\infty} J_{2 n-1}(m)\left\{(-1)^{n} \cos \left[(2 n-1) \omega_{R F} t\right]-\sin \left[(2 n-1) \omega_{R F} t\right]\right\} \\
-i \sum_{n=1}^{\infty} J_{2 n-1}(m)\left\{(-1)^{n} \cos \left[(2 n-1) \omega_{R F} t\right]-i \sin \left[(2 n-1) \omega_{R F} t\right]\right\}
\end{array}\right]\left[I_{1}\right]} \\
& {\left[\begin{array}{c}
O_{1} \\
O_{2} \\
O_{3} \\
O_{4}
\end{array}\right]=\frac{1}{2}\left[\begin{array}{c}
i\left\{J_{1}(m) \exp \left[-j \omega_{R F} t\right]-J_{3}(m) \exp \left[j 3 \omega_{R F} t\right] \ldots\right\} \\
\sqrt{2} e^{i 3 \pi / 4}\left\{-J_{1}(m) \cos \left(\omega_{R F} t+\pi / 4\right)+J_{3}(m) \cos \left(3 \omega_{R F} t-\pi / 4\right)\right\} \\
\sqrt{2} e^{i 3 \pi / 4}\left\{J_{1}(m) \cos \left(\omega_{R F} t-\pi / 4\right)-J_{3}(m) \cos \left(3 \omega_{R F} t+\pi / 4\right)\right\} \\
i\left\{J_{1}(m) \exp \left[j \omega_{R F} t\right]-J_{3}(m) \exp \left[-j 3 \omega_{R F} t\right] \ldots\right\}
\end{array}\right]\left[I_{1}\right]}
\end{aligned}
$$


It can be seen from equation (8) that frequency shifting utilizing the SSB modulation in one IQ modulator can be obtained by the proposed two parallel IQ modulator architecture. Removing a part of the circuit from the operation without physically disconnecting it offers a valuable degree of freedom in any photonic integrating architecture. Equation (8) verifies that apart from the optical loss, the reduced circuit can still offer electric to optical frequency up-conversion with the same spatial specification and spectral purity.

It can be observed from equation (7) and (8) that the lower and upper optical first-order harmonics are conveniently spatially separated and available from output port $O_{1}$ and $O_{4}$ when only input port $I_{1}$ is coupled to the optical source. The $3^{\text {rd }}$ order harmonics can be suppressed by properly selecting the RF amplitude. This facilitates remote heterodyning for wireless access network using a digital coherent RoF system. One of the two harmonics can be modulated by a second IQ modulator and the two harmonics combined and propagated through the optical fiber. After its transmission to a high-speed photodetector at the remote antenna, the two harmonics beat to produce a modulated RF carrier with a center frequency equal to the double of the RF frequency. The multiplication factor is small and so, high RF frequency is needed to access the millimeter-wave regime.

\section{Device fabrication:}

(a)

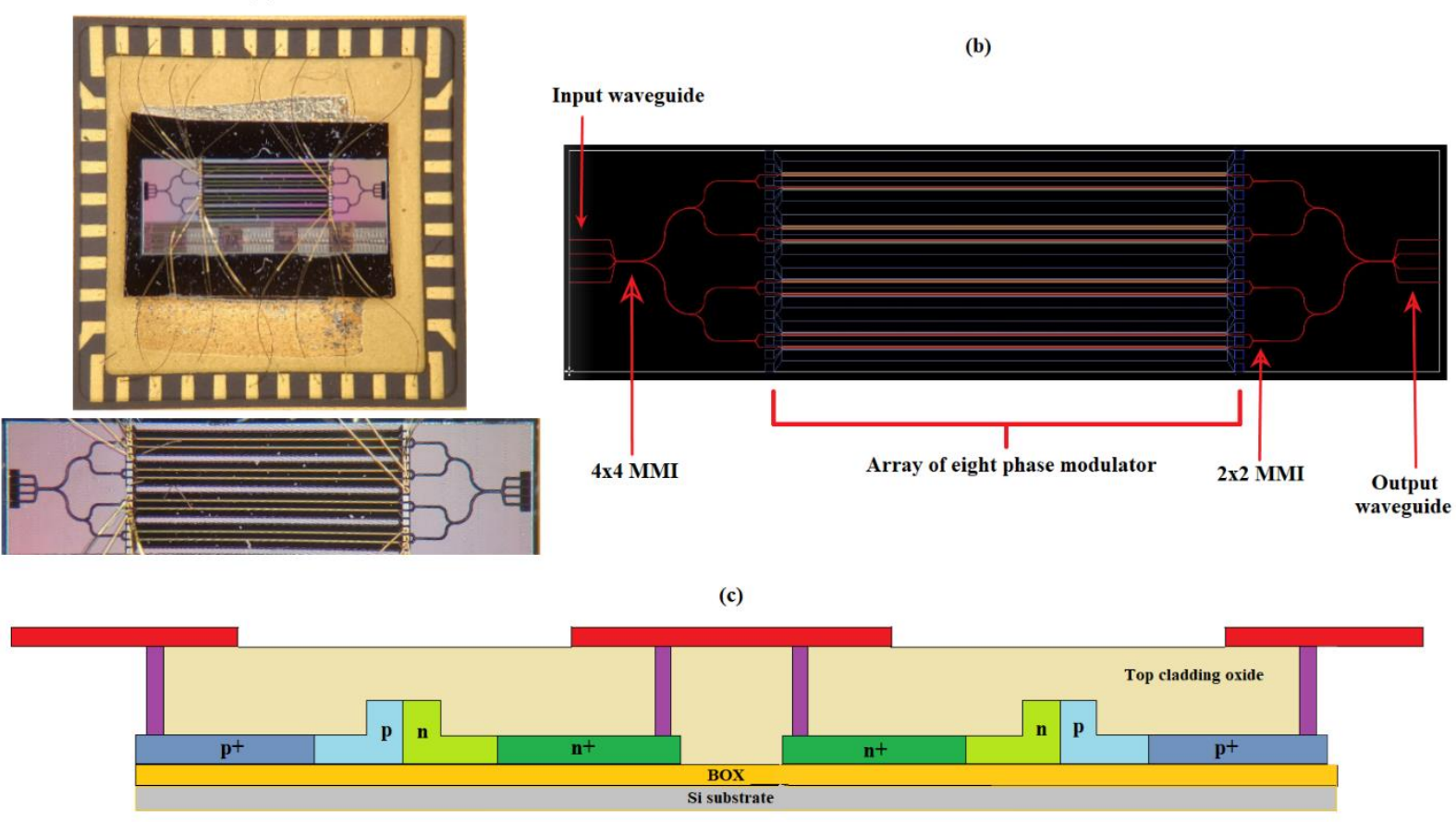

Figure 3: (a) Top-view microscopic image of the fabricated chip; (b) Mask layout of the device; (c) Cross-sectional diagram of the MZI arm

Figure 3(a) and 3(b) show the top-view microscope image and the mask layout of the frequency upconverter. Device fabrication was performed using the A*Star Institute of Microelectronics (IME) CMOS compatible process on a SOI wafer. The SOI wafer has a top Si thickness of $220 \mathrm{~nm}$ and buried oxide thickness of $2 \mu \mathrm{m}$ [54]. The circuit consists of four $2 \times 2$ MMI-MZMs connected in parallel in between two $4 \times 4$ MMIs. The large width of the electro-optic modulator stack in comparison to the $\mathrm{I} / \mathrm{O}$-end-MMIs introduces bends. So, path-length matching becomes a critical design point. From figure 3(b), it can be observed that racetrack method has been applied where concentric circles are used to ensure that each lane encounters compensating short and long paths around bends. The absence of any mechanism for efficient 
coupling of light to and from the circuit at the edges of the chip is the primary limiter of functionality in terms of insertion loss.

Each intensity modulator is a Mach-Zehnder interferometer (MZI) with a reverse biased p-n diode structure embedded in each of the two arms. The p-n junction is formed at the middle of the Si rib waveguide between lightly doped $\mathrm{p}$ and $\mathrm{n}$ regions which extend to the slab. Figure 3(c) shows the cross-sectional schematic of the MZI modulation arms. To ensure good ohmic contact between silicon and metal contacts, two heavily doped slab regions are placed away from both sides of the rib edge. The additional optical loss due to absorption in the heavily doped region can be taken as negligible due to the choice of rib height-slab thickness ratio [54]. After doping, the implanted dopants were activated using a rapid thermal anneal (RTA) at $1030^{\circ} \mathrm{C}$ for 5 seconds. Two level metal system is used for interconnect. Travelling wave electrode based on coplanar waveguide structure are formed for the input of modulating signal. No termination impedance have been fabricated on the chip or applied in the experiment.

\section{Experimental result and discussion:}

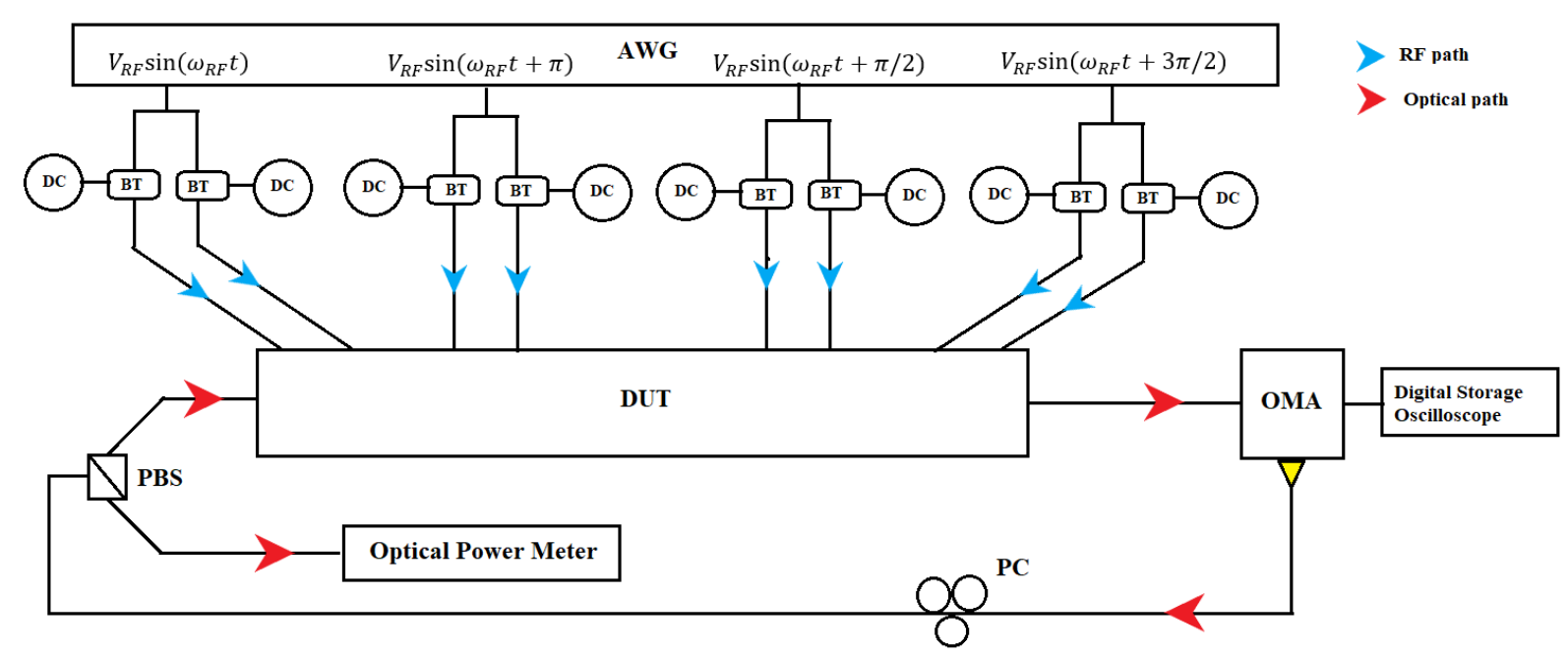

Figure 4: Schematic of the measurement setup. The built-in laser diode of the optical modulation analyzer (OMA) is used as the input light source to maintain better coherency at the receiver side. Light is coupled to the access waveguide at the input/output ends of the chip utilizing lensed polarization maintaining fiber. DC sources are connected to the RF signals generated from arbitrary waveform generator (AWG) via bias tees (BT). Polarization controller (PC) and polarization beam splitter (PBS) are used so that only TE is transmitted through the chip. Optical power meter is to observe the stability of the input polarization state.

The setup used for experimental demonstration of the phase shifter is sketched in figure 4 . The built-in laser diode of an optical modulation analyzer (Agilent N4391A) is used as the optical source. To maintain polarization of the light input to the silicon chip to be TE, a polarization controller (PC) followed by a polarization beam-splitter (PBS) is connected between the light source and the DUT. An optical power meter (Anritsu ML910B) is used to measure the power of TM component. By adjusting the PC, the TM component can be minimized and the stability of the polarization state of the input light can be observed and maintained. Lensed fibers are used for input-output coupling. The output of the silicon chip is fed back to the OMA which is connected to the digital storage oscilloscope (Infiniium DSO-20-91604A). Polarization maintaining fiber is used for all connections.

Static assessment: To isolate individual MZI's DC characteristic, it is imperative that the other MZIs on the parallel structure are perfectly balanced and biased at their null point. The $4 \times 4$ MMIs at the edges should also be balanced in terms of splitting ratio and phase relationship. Ideally, from the structure shown in figure 2(a), the MZIs are biased at their null point by design and due to the equal path length between the internal $2 \times 2$ MMIs, they are acting as a cross-over in the absence of any outside perturbation. But any minute width 
deviation in the high contrast SOI platform due to fabrication tolerance or poor design can create huge phase error. So in practice, for SOI one might as well assume every MZI is randomly unbalanced.

(a) MZM

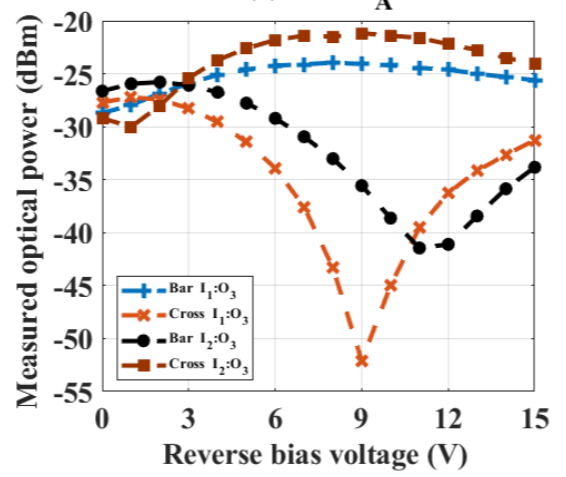

(b) MZM $_{\text {B }}$

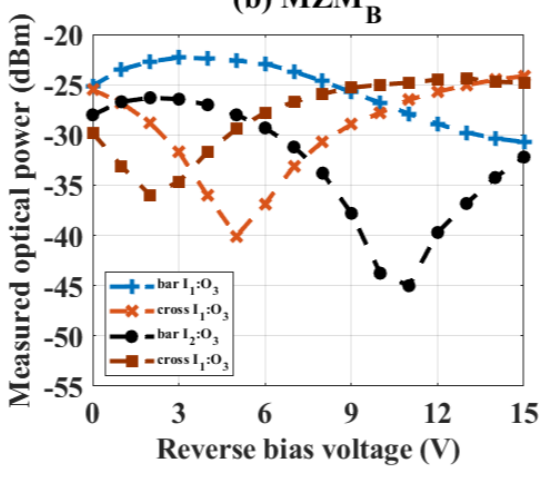

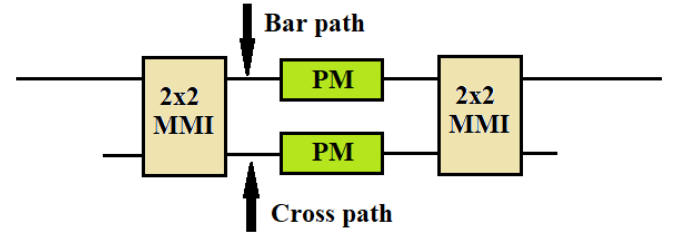

Figure 5: DC characteristics of the MZMs $\left(M_{A} M_{A}\right.$ and $\left.M Z M_{B}\right)$ in terms of output optical power due to the variation of reverse bias voltage. Input port $1 \& 2$ are considered for light input. Output is taken from output port 3. Each MZM is asymmetrically driven which means reverse bias is applied to only one arm of the corresponding MZM: Reverse bias voltage is applied to only (a) $\mathrm{MZM}_{\mathrm{A}}$ and (b) $\mathrm{MZM}_{\mathrm{B}}$

Figure 5 shows the variation of optical output power due to the reverse bias voltage change. Two MZMs $\left(\mathrm{MZM}_{\mathrm{A}}\right.$ and $\left.\mathrm{MZM}_{\mathrm{B}}\right)$ are considered for the DC test and two input-output port combinations $\left(\mathrm{I}_{1} \rightarrow \mathrm{O}_{3}\right.$ and $\mathrm{I}_{2} \rightarrow \mathrm{O}_{3}$ ) are taken. It can be observed from figure 5(a) that over the range of the bias voltage, the notch in the transmission (minimum transmission) for biasing one arm of $\mathrm{MZM}_{\mathrm{A}}$ corresponds to a flat peak transmission (maximum) for biasing the other arm. This can be expected as the light propagating through the cross arm is subjected to a total $\pi$ phase shift. Their roles have been interchanged with noteworthy deviations when the input condition was changed. Similar behaviour can be observed for $\mathrm{MZM}_{\mathrm{B}}$ as shown in figure 5(b), although there are obvious shifts in its responses relative to $\mathrm{MZM}_{\mathrm{A}}$. These data suggest that biasing of the MZMs in the device needs individual fine tuning. In addition, the contributions of the offstate, imbalanced MZMs cannot be neglected.

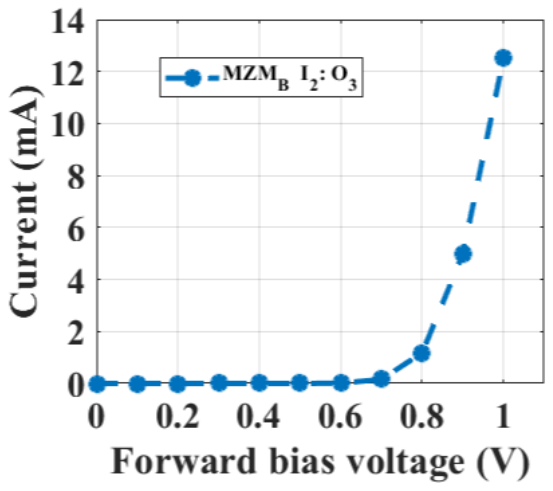

Figure 6: Measured forward bias I-V characteristic of a phase modulator on MZMB 
Figure 6 shows the forward bias I-V characteristic of a phase modulator in one arm of MZM measurement can be found in $[50,53]$ which determines that the diode performance has not deteriorated over the years.

Dynamic assessment: Individual characterization of the MZMs continued with the application of RF input. An arbitrary waveform generator (Fluke 294) with sinusoid frequency limited to $40 \mathrm{MHz}$ is used as the RF source. $\mathrm{MZM}_{\mathrm{B}}$ is driven differentially. Two channels from the arbitrary waveform generator (AWG) provide two $10 \mathrm{MHz}$ sinusoidal signals with one is $\pi$ phase shifted relative to the other. The RF amplitude has not been adjusted to maximize the desired harmonic. The output optical spectrum is shown in figure 7. The horizontal axis is the relative frequency with respect to the optical frequency of the input light and the vertical axis is the optical spectrum received at the OMA. The carrier frequency is $193.4125 \mathrm{THz}$ and the resolution bandwidth of the OMA is $477.42 \mathrm{kHz}$. In an ideal scenario, a differentially driven MZM biased

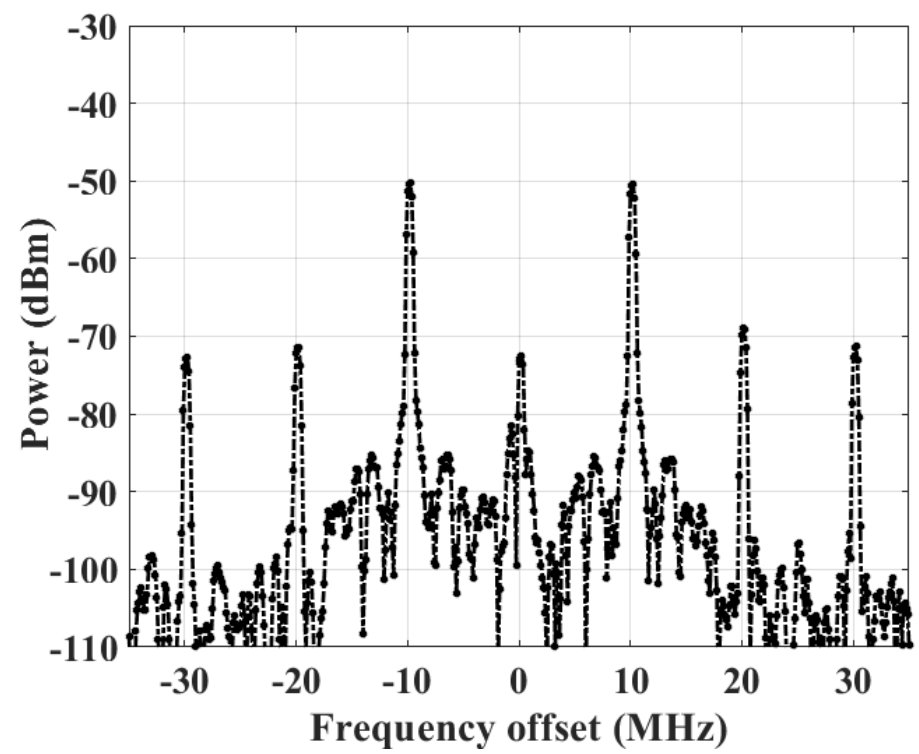

Figure 7: Optical spectrum of the circuit when only one MZM (MZMв) is modulating.

at its MITP acts as an intensity modulator. For a pure tone modulating signal, carrier and even order harmonics are completely suppressed where harmonics are spaced by the value of RF frequency. The odd harmonics remain with intensity weighted by Bessel functions of modulation index with the same order as the harmonic. Figure 7 shows deviation from the ideal case. The carrier and $2^{\text {nd }}$ order harmonics are present which reflect the fact that other MZMs are contributing to the carrier breakthrough. The imbalance in the $\mathrm{MZM}_{\mathrm{B}}$ results the imperfect suppression of the $2^{\text {nd }}$ or any other even order harmonics. The dual-drive configuration can be exploited so that individual adjustment of the modulation voltage of each phase shifter can be performed to suppress the unwanted harmonics.

Appropriate RF outputs from the AWG are applied to the frequency shifter circuit, as shown in figure 4. Light is launched into the chip through input port 1 and outputs are taken from port 1 and 4 . The optical spectrums are shown in figure 8 . It can be observed that frequency up-conversion has been achieved. Figure 8(a) and 8(b) represent the LSB and USB modulation based frequency shifting operation. The theoretically pristine performance of the circuit is limited by the presence of carrier and undesired spurious sidebands. A carrier suppression of $\sim 20 \mathrm{~dB}$ and lowest spurious harmonic suppression of $\sim 12 \mathrm{~dB}$ relative to the desired harmonic have been measured. 
(a)

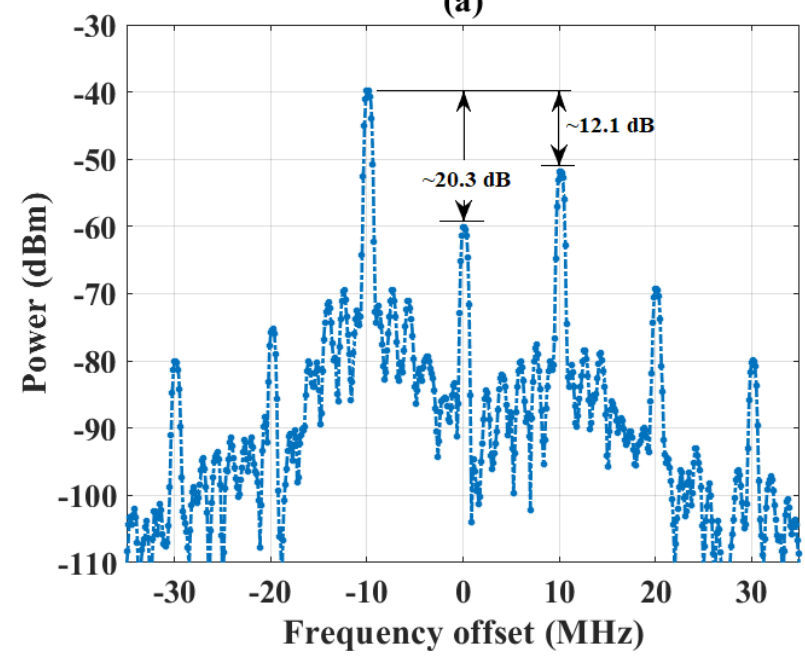

(b)

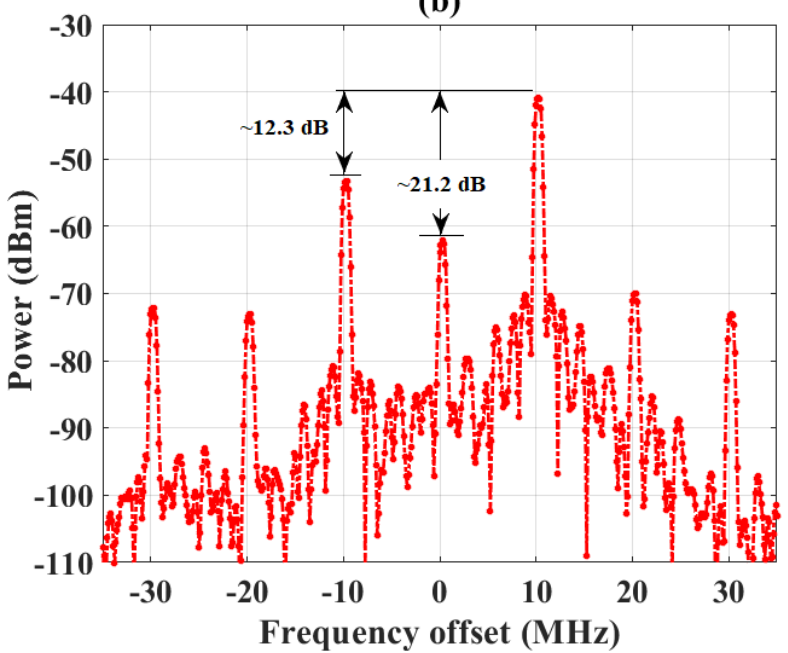

Figure 8: Optical spectra representing frequency shifting functionality of the fabricated circuit. (a) Frequency down-conversion at outport port 1 and (b) frequency up-conversion at output port 4 . The resolution bandwidth of the optical modulation analyzer is $668.388 \mathrm{kHz}$

From the optical spectra of the frequency shifter, it can be concluded that the finite extinction ratio of the MZMs, power imbalance and phase errors between the ports of the MMIs are imposing the deviations from the ideal situation. Mehedi et al. have analyzed the performance of the circuit by simulation considering these impairments [50]. They modelled their MMIs based on the results obtained in [55]. By comparing their simulation results and experimental data obtained here, it can be estimated that the impairments due to the fabrication errors are more severe than simulated. The only tuning mechanism adopted in our experiment is the adjustment of DC bias voltage. Further tuning can be done at fabrication level by incorporating additional phase shifter [56] or integrated variable coupler and variable attenuator [19] components. In addition, Miller described a self-adjustment approach to compensate for imperfect extinction ratio which could be adopted to minimize amplitude and phase imbalance [57]. Furthermore, broadband sub-wavelength engineered MMI offers reduced phase error and power imbalance which could be a viable solution [58-59].

Another important observation is the high insertion loss $(\sim 20-30 \mathrm{~dB})$. The principle reason is the absence of any mechanism for matching between the fiber mode and high-contrast access guide mode which could lead huge coupling loss. The lack of test structure on the chip prevents the author to measure insertion loss of individual component. Because of the high contrast material platform and relatively large sidewall roughness of the narrow ridges, on-chip waveguide optical loss may be relatively high $(\sim 2-6 \mathrm{~dB})$. The MMIs and doped phase shifters may contribute similar loss. From these practical assumption, it can be conjectured that the principle contributor of the insertion loss is the fiber-chip coupling ( 12-14 dB). It is also observed that the loss varies depending upon the choice of input-output port combination. Several defects have been observed on the chip. Figure 9 shows a defect at one of the access guide which shows the highest loss. It is imperative that for future fabrication, the access guides should have proper coupling mechanism. A variety of edge coupling schemes for low-loss coupling between the optical fiber and silicon photonic wire on high-contrast material platform have been proposed which can be adopted [60-61]. In addition to the structural issues, the RF amplitude has not been tuned to critically adjust the modulation index of each modulator and thus maximize $1^{\text {st }}$ order harmonics at the output. 


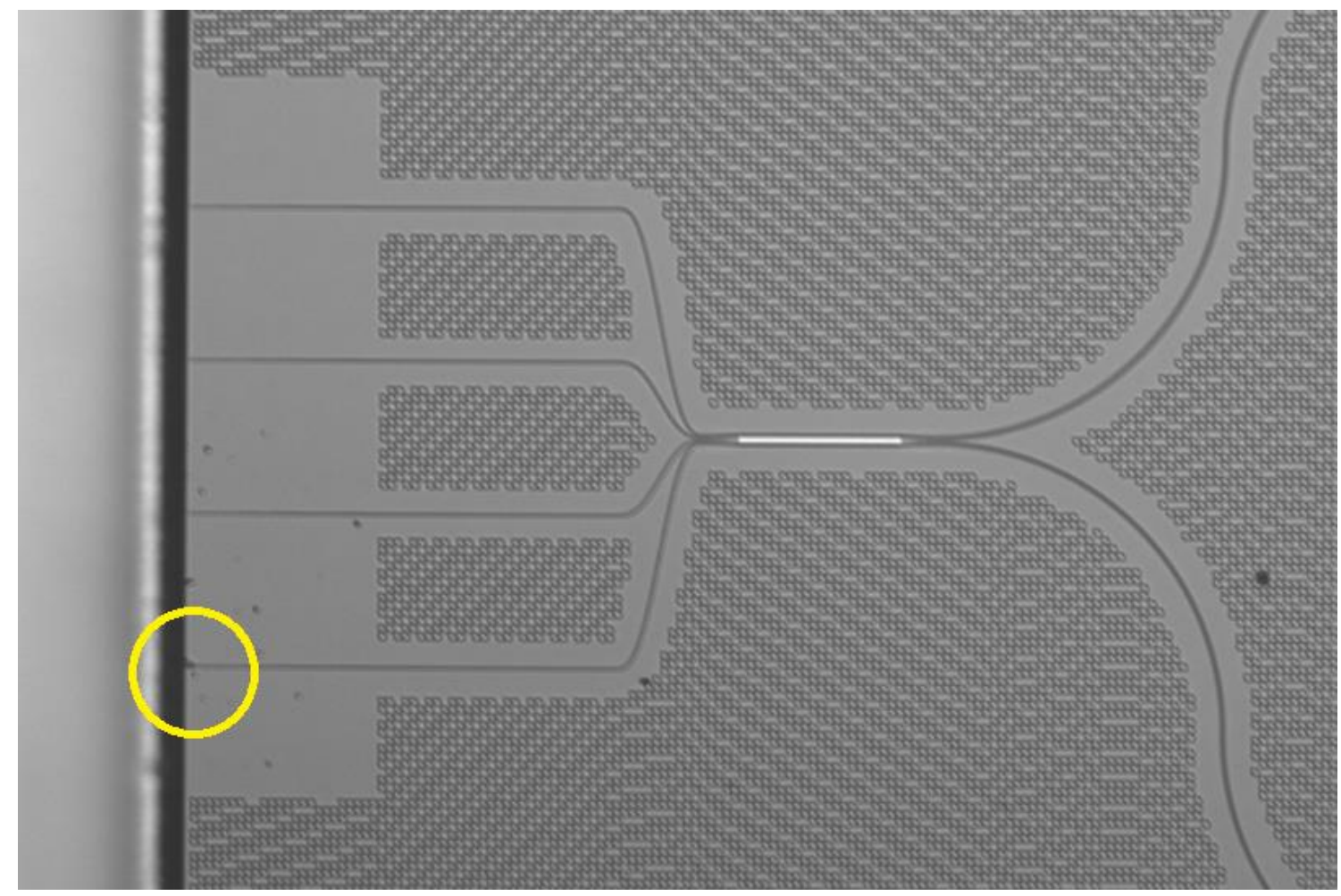

Figure 9: Microscopic image of the defect at the edge-end of the access waveguide

\section{Conclusion:}

In summary, we have demonstrated an optical SSB modulation based frequency shifter on SOI platform and its performance has been evaluated. A carrier suppression ratio of $\sim 20 \mathrm{~dB}$ and spurious sideband suppression ratio of $\sim 12 \mathrm{~dB}$ have been achieved by only limited DC bias tuning. No additional imbalance compensating mechanism has been adopted. The quality of the operation can be improved by reducing fabrication errors, optimizing the phase shifter and MMI design and implementing integrated edge coupling and imbalance compensating technique. Although only low RF frequency operation is being reported due to the limit imposed by equipment, this device should be capable of operating in the full bandwidth of the modulators $(\sim 10 \mathrm{GHz})$.

The architecture is a multi-functional device which offers operations other than optical frequency shifting. Complex modulation, frequency multiplication for millimeter-wave application and sub-carrier generation to facilitate high data rate transmission using OFDM technology can be achieved without any modification inside the circuit. The DC bias less design with inherently off-state MZM in parallel offers freedom to choose any modulator and flexibility to exploit and further extend its functionality. The demonstrated circuit has been fabricated using 'off-the-shelf' components as a proof of implementation feasibility. There is still scope for improvements in terms of component design and state-of-the-art fabrication to achieve compact device with low loss, low power, spectrally pure and high bandwidth operation. In addition to SOI, the circuit can be fabricated in any other material platform offering electro-optic modulator such as $\mathrm{LiNbO}_{2}$, III-V or hybrid technology to exploit the advantages they render.

\section{Acknowledgement:}

The fabrication of the circuit was supported by CMC microsystem. The authors would like to show their gratitude to Dr. Jessica Zhang, Engineering consultant - photonics at CMC Microsystems for her contribution to mask layout. The authors also thank Dr. Ramón Maldonado-Basilio for his help in wire 
bonding. Mehedi Hasan acknowledges the Natural Sciences and Engineering Research Council of Canada (NSERC) for their support through the Vanier Canada Graduate Scholarship program. Trevor Hall is grateful to the University of Ottawa for their support of his University Research Chair.

\section{Reference:}

1. Kazovsky, Leonid G., Wei-Tao Shaw, David Gutierrez, Ning Cheng, and Shing-Wa Wong. "Nextgeneration optical access networks." Journal of lightwave technology 25, no. 11 (2007): 34283442 .

2. Gaudino, Roberto, Daniel Cárdenas, Martial Bellec, Benoît Charbonnier, Noëlla Evanno, Philippe Guignard, Sylvain Meyer, Anna Pizzinat, Ingo Mollers, and Dieter Jager. "Perspective in nextgeneration home networks: Toward optical solutions?." IEEE Communications Magazine 48, no. 2 (2010): 39-47.

3. Cox, Charles H., Edward I. Ackerman, Gary E. Betts, and Joelle L. Prince. "Limits on the performance of RF-over-fiber links and their impact on device design." IEEE Transactions on Microwave Theory and Techniques 54, no. 2 (2006): 906-920.

4. Decena, Bernalyn, Marc Rosales, and Paul Jason Co. "Characterization of Low Cost Wideband Direct Modulation Radio over Fiber Link." In 2019 International Symposium on Multimedia and Communication Technology (ISMAC), pp. 1-5. IEEE, 2019.

5. Hall TJ, Maldonado-Basilio R, Abdul-Majid S, Seregelyi J, Li R, Antolín-Pérez I, Nikkhah H, Lucarz F, de La JL, Fracasso B, Pajusco P. Radio-over-Fibre access for sustainable Digital Cities. annals of telecommunications-annales des télécommunications. 2013 Feb 1;68(1-2):3-21.

6. Alavi, S. E., M. R. K. Soltanian, I. S. Amiri, M. Khalily, A. S. M. Supa'At, and Harith Ahmad. "Towards 5G: A photonic based millimeter wave signal generation for applying in 5G access fronthaul." Scientific reports 6, no. 1 (2016): 1-11.

7. Chen, Xiang, and Jianping Yao. "A high spectral efficiency coherent rof system based on OSSB modulation with low-cost free-running laser sources for UDWDM-PONs." Journal of Lightwave Technology 34, no. 11 (2016): 2789-2795.

8. Hirooka, Toshihiko, Masato Yoshida, Keisuke Kasai, and Masataka Nakazawa. "Optical and wireless-integrated next-generation access network based on coherent technologies." In Broadband Access Communication Technologies X, vol. 9772, p. 977203. International Society for Optics and Photonics, 2016.

9. Kim, Cheolhwan, Inwoong Kim, Guifang Li, Michael R. Lange, Timothy E. Dimmick, Patrick Langlois, and Benoit Reid. "Optical microwave/millimeter-wave links using direct modulation of two-section gain-coupled DFB lasers." IEEE photonics technology letters 17, no. 8 (2005): 17341736.

10. Li, Yifei, Maja Bystrom, David Yoo, Samuel M. Goldwasser, and Peter R. Herczfeld. "Coherent optical vector modulation for fiber radio using electrooptic microchip lasers." IEEE transactions on microwave theory and techniques 53, no. 10 (2005): 3121-3129.

11. Doi, Yoshiyuki, Seiji Fukushima, Tetsuichiro Ohno, and Kaoru Yoshino. "Frequency stabilization of millimeter-wave subcarrier using laser heterodyne source and optical delay line." IEEE Photonics Technology Letters 13, no. 9 (2001): 1002-1004.

12. Kuri, Toshiaki, and K-I. Kitayama. "Long-term stabilized millimeter-wave generation using a highpower mode-locked laser diode module." IEEE transactions on microwave theory and techniques 47, no. 5 (1999): 570-574.

13. O'reilly, J. J., P. M. Lane, Rolf Heidemann, and Rolf Hofstetter. "Optical generation of very narrow linewidth millimetre wave signals." Electronics Letters 28, no. 25 (1992): 2309-2311.

14. Hasan, Gazi Mahamud, Mehedi Hasan, Hongpeng Shang, DeGui Sun, Karin Hinzer, Peng Liu, and Trevor Hall. "Energy efficient photonic millimeter-wave generation using cascaded polarization modulators." Optical and Quantum Electronics 51, no. 7 (2019): 217. 
15. Hasan, Gazi Mahamud, Mehedi Hasan, Karin Hinzer, and Trevor Hall. "Optical frequency multiplication technique using cascaded modulator to achieve RF power advantage." In 2019 International Conference on Numerical Simulation of Optoelectronic Devices (NUSOD), pp. 125126. IEEE, 2019.

16. Inoue, Kyo, Norio Takato, Hiromu Toba, and Masao Kawachi. "A four-channel optical waveguide multi/demultiplexer for 5-GHz spaced optical FDM transmission." Journal of lightwave technology 6, no. 2 (1988): 339-345.

17. Toda, Hiroyuki, Tsukasa Yamashita, Toshiaki Kuri, and Ken-ichi Kitayama. "Demultiplexing using an arrayed-waveguide grating for frequency-interleaved DWDM millimeter-wave radio-onfiber systems." Journal of lightwave technology 21, no. 8 (2003): 1735-1741.

18. Yamazaki, Hiroshi, Takashi Saida, Takashi Goh, Atsushi Mori, and Shinji Mino. "Dual-carrier IQ modulator with a complementary frequency shifter." Optics express 19, no. 26 (2011): B69-B74.

19. Yamazaki, H.; Saida, T.; Goh, T.; Mino, S.; Nagatani, M.; Nosaka, H.; Murata, K. Dual-carrier Dual-polarization IQ Modulator Using a Complementary Frequency Shifter. IEEE J. Sel. Top. Quant. Electron. 2013, 19 (6), 3400208.

20. Hasan, Mehedi, and Trevor J. Hall. "Photonic circuit for high order USB and LSB separation for remote heterodyning: analysis and simulation." Optics express 23, no. 19 (2015): 25259-25271.

21. Dorrington, Adrian A., and Rainer Kuennemeyer. "Single sideband techniques for laser Doppler velocimeter frequency offset." Optical Engineering 42, no. 11 (2003): 3239-3247.

22. Li, Yanlu, Stijn Meersman, and Roel Baets. "Optical frequency shifter on SOI using thermo-optic serrodyne modulation." In 7th IEEE International Conference on Group IV Photonics, pp. 75-77. IEEE, 2010.

23. Li, Jianping, and Zhaohui Li. "Frequency-locked multicarrier generator based on a complementary frequency shifter with double recirculating frequency-shifting loops." Optics letters 38, no. 3 (2013): 359-361.

24. Li, Jianping, Xuebing Zhang, Zhaohui Li, Xiaoguang Zhang, Guifang Li, and Chao Lu. "Theoretical studies on the polarization-modulator-based single-side-band modulator used for generation of optical multicarrier." Optics express 22, no. 12 (2014): 14087-14095.

25. Kowalski, F. V., P. D. Hale, and S. J. Shattil. "Broadband continuous-wave laser." Optics letters 13, no. 8 (1988): 622-624.

26. Frankel, Michael Y., R. D. Esman, and M. G. Parent. "Array transmitter/receiver controlled by a true time-delay fiber-optic beamformer." IEEE Photonics Technology Letters 7, no. 10 (1995): 1216-1218.

27. Cygan, Agata, Daniel Lisak, Piotr Morzyński, Marcin Bober, Michał Zawada, Eugeniusz Pazderski, and Roman Ciuryło. "Cavity mode-width spectroscopy with widely tunable ultra narrow laser." Optics express 21, no. 24 (2013): 29744-29754.

28. Tanabe, Takasumi, M. Notomi, and E. Kuramochi. "Measurement of ultra-high-Q photonic crystal nanocavity using single-sideband frequency modulator." Electronics letters 43, no. 3 (2007): 187 188.

29. Shagam, Richard N., and James C. Wyant. "Optical frequency shifter for heterodyne interferometers using multiple rotating polarization retarders." Applied optics 17, no. 19 (1978): 3034-3035.

30. Nosu, K., S. C. Rashleigh, H. F. Taylor, and J. F. Weller. "Acousto-optic frequency shifter for single-mode fibres." Electronics Letters 19, no. 20 (1983): 816-818.

31. Cummins, H. Z., and N. Knable. "Single sideband modulation of coherent light by Bragg reflection from acoustical waves." Proceedings of the IEEE 51, no. 9 (1963): 1246-1246.

32. Izutsu, Masayuki, Shinsuke Shikama, and Tadasi Sueta. "Integrated optical SSB modulator/frequency shifter." IEEE Journal of Quantum Electronics 17, no. 11 (1981): 2225-2227. 
33. Hui, Rongqing, Benyuan Zhu, Renxiang Huang, Christopher T. Allen, Kenneth R. Demarest, and Douglas Richards. "Subcarrier multiplexing for high-speed optical transmission." Journal of lightwave technology 20, no. 3 (2002): 417.

34. Frankel, Michael Y., and Ronald D. Esman. "Optical single-sideband suppressed-carrier modulator for wide-band signal processing." Journal of lightwave technology 16, no. 5 (1998): 859.

35. Xiao, Shijun, and Andrew M. Weiner. "Optical carrier-suppressed single sideband (O-CS-SSB) modulation using a hyperfine blocking filter based on a virtually imaged phased-array (VIPA)." IEEE photonics technology letters 17, no. 7 (2005): 1522-1524.

36. Zibar, Darko, Rakesh Sambaraju, Antonio Caballero Jambrina, Ruben Alemany, Javier Herrera, and Idelfonso Tafur Monroy. "16 Gb/s QPSK Wireless-over-Fibre link in $75-110 \mathrm{GHz}$ band employing optical heterodyne generation and coherent detection." In 36th European Conference and Exhibition on Optical Communication, pp. 1-3. IEEE, 2010.

37. Shimotsu, S., S. Oikawa, T. Saitou, N. Mitsugi, K. Kubodera, T. Kawanishi, and M. Izutsu. "Single side-band modulation performance of a $\mathrm{LiNbO}_{3}$ integrated modulator consisting of four-phase modulator waveguides." IEEE Photonics Technology Letters 13, no. 4 (2001): 364-366.

38. Yamaguchi, Yuya, Atsushi Kanno, Tetsuya Kawanishi, Masayuki Izutsu, and Hirochika Nakajima. "Pure single-sideband modulation using high extinction-ratio parallel Mach-Zehnder modulator with third-order harmonics superposition technique." In 2015 Conference on Lasers and ElectroOptics (CLEO), pp. 1-2. IEEE, 2015.

39. Yu, Jianjun, Zhensheng Jia, Lilin Yi, Yikai Su, Gee-Kung Chang, and Ting Wang. "Optical millimeter-wave generation or up-conversion using external modulators." IEEE Photonics technology letters 18, no. 1 (2005): 265-267.

40. Chow, C. W., C. H. Wang, C. H. Yeh, and S. Chi. "Analysis of the carrier-suppressed singlesideband modulators used to mitigate Rayleigh backscattering in carrier-distributed PON." Optics express 19, no. 11 (2011): 10973-10978.

41. Lauermann, Matthias, Claudius Weimann, A. Knopf, Wolfgang Heni, Robert Palmer, Sebastian Koeber, Delwin L. Elder et al. "Integrated optical frequency shifter in silicon-organic hybrid (SOH) technology." Optics express 24, no. 11 (2016): 11694-11707.

42. Kodigala, A., M. Gehl, C. T. DeRose, D. Hood, A. T. Pomerene, C. Dallo, D. Trotter et al. "Silicon Photonic Single-Sideband Generation with Dual-Parallel Mach-Zehnder Modulators." In 2019 Conference on Lasers and Electro-Optics (CLEO), pp. 1-2. IEEE, 2019.

43. Heismann, F., and R. Ulrich. "Integrated-optical frequency translator with stripe waveguide." Applied physics letters 45, no. 5 (1984): 490-492.

44. Johnson, Leonard M., and Ch H. Cox. "Serrodyne optical frequency translation with high sideband suppression." Journal of lightwave technology 6, no. 1 (1988): 109-112.

45. Li, Yanlu, Stijn Meersman, and Roel Baets. "Optical frequency shifter on SOI using thermo-optic serrodyne modulation." In 7th IEEE International Conference on Group IV Photonics, pp. 75-77. IEEE, 2010.

46. Barretto, Elaine CS, and Jørn M. Hvam. "Photonic integrated single-sideband modulator/frequency shifter based on surface acoustic waves." In Silicon photonics and photonic integrated circuits II, vol. 7719, p. 771920. International Society for Optics and Photonics, 2010.

47. Fan, Guofang, Yuan Li, Chunguang Hu, Lihua Lei, Dong Zhao, Hongyu Li, and Zhen Zhen. "A novel concept of acousto-optic ring frequency shifters on silicon-on-insulator technology." Optics \& Laser Technology 63 (2014): 62-65.

48. Shen, Yichun, Xianmin Zhang, and Kangsheng Chen. "Optical single sideband modulation of 11GHz RoF system using stimulated Brillouin scattering." IEEE photonics technology letters 17, no. 6 (2005): 1277-1279.

49. Sharma, Gourav Prateek, Stefan Preußler, and Thomas Schneider. "Precise optical frequency shifting using stimulated Brillouin scattering in optical Fibers." IEEE Photonics Technology Letters 29, no. 17 (2017): 1467-1470. 
50. Hasan, Mehedi, Jianqi Hu, Hamdam Nikkhah, and Trevor Hall. "A photonic circuit for complementary frequency shifting, in-phase quadrature/single sideband modulation and frequency multiplication: analysis and integration feasibility." Journal of Modern Optics 64, no. 14 (2017): 1386-1397.

51. Maldonado-Basilio, Ramón, Mehedi Hasan, Hamdam Nikkhah, Sawsan Abdul-Majid, Rabiaa Guemri, Frédéric Lucarz, Jean-Louis de Bougrenet de la Tocnaye, and Trevor James Hall. "Electrooptic up-conversion mixer amenable to photonic integration." Journal of Modern Optics 62, no. 17 (2015): 1405-1411.

52. Mehedi Hasan, Rabiaa Guemri, Ramón Maldonado-Basilio, Frédéric Lucarz, Jean-Louis de Bougrenet de la Tocnaye, and Trevor Hall, " Theoretical analysis and modeling of a photonic integrated circuit for frequency 8-tupled and 24-tupled millimeter wave signal generation", OPTICS LETTERS / Vol. 39, No. 24 / December 15, 2014

53. Maldonado-Basilio, Ramón, Neda Nabavi, Kaveleen Aulakh, Mehedi Hasan, and Trevor J. Hall. "Photonic integrated circuit for millimeter-wave signal generation by frequency multiplication." In 2015 IEEE Photonics Conference (IPC), pp. 130-131. IEEE, 2015.

54. Liow, Tsung-Yang, Kah-Wee Ang, Qing Fang, Jun-Feng Song, Yong-Zhong Xiong, Ming-Bin Yu, Guo-Qiang Lo, and Dim-Lee Kwong. "Silicon modulators and germanium photodetectors on SOI: monolithic integration, compatibility, and performance optimization." IEEE Journal of Selected Topics in Quantum Electronics 16, no. 1 (2009): 307-315.

55. Abdul-Majid, Sawsan, Ramón Maldonado-Basilio, Chengmin Lei, Hazem Awad, Imad Hasan, N. Ye Winnie, and Trevor J. Hall. "Performance analysis of a photonic integrated interferometer circuit based on silicon-on-insulator." Optical and Quantum Electronics 47, no. 7 (2015): 19651971.

56. Kodigala, A., M. Gehl, C. T. DeRose, D. Hood, A. T. Pomerene, C. Dallo, D. Trotter et al. "Silicon Photonic Single-Sideband Generation with Dual-Parallel Mach-Zehnder Modulators." In 2019 Conference on Lasers and Electro-Optics (CLEO), pp. 1-2. IEEE, 2019.

57. Miller, David AB. "Perfect optics with imperfect components." Optica 2, no. 8 (2015): 747-750.

58. Ortega-Moñux A, Alonso-Ramos C, Maese-Novo A, Halir R, Zavargo-Peche L, Pérez-Galacho D, Molina-Fernández I, Wangüemert-Pérez JG, Cheben P, Schmid JH, Lapointe J. An ultra-compact multimode interference coupler with a subwavelength grating slot. Laser \& Photonics Reviews. 2013 Mar;7(2):L12-5.

59. Halir R, Bock PJ, Cheben P, Ortega-Moñux A, Alonso-Ramos C, Schmid JH, Lapointe J, Xu DX, Wangüemert-Pérez JG, Molina-Fernández Í, Janz S. Waveguide sub-wavelength structures: a review of principles and applications. Laser \& Photonics Reviews. 2015 Jan;9(1):25-49.

60. Cheben, Pavel, Jens H. Schmid, Shurui Wang, Dan-Xia Xu, Martin Vachon, Siegfried Janz, Jean Lapointe, Yves Painchaud, and Marie-Josée Picard. "Broadband polarization independent nanophotonic coupler for silicon waveguides with ultra-high efficiency." Optics express 23, no. 17 (2015): 22553-22563.

61. Picard, M-J., Y. Painchaud, C. Latrasse, C. Larouche, F. Pelletier, and M. Poulin. "Novel spot-size converter for optical fiber to sub- $\mu \mathrm{m}$ silicon waveguide coupling with low loss, low wavelength dependence and high tolerance to alignment." In 2015 European Conference on Optical Communication (ECOC), pp. 1-3. IEEE, 2015. 
